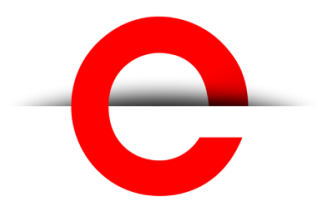

U T S

e PRES S

\section{PORTAL Journal of}

Multidisciplinary

International Studies

Vol. 14, No. 1

April 2017

Transitions and Dislocations, Curated Cultural Works Issue, Curated by Paul Allatson.

(C) 2017 by Susana ChávezSilverman. This is an Open Access article distributed under the terms of the Creative Commons Attribution 4.0 Unported (CC BY 4.0) License (https:// creativecommons.org/ licenses/by/4.0/l, allowing third parties to copy and redistribute the material in any medium or format and to remix, transform, and build upon the material for any purpose, even commercially, provided the original work is properly cited and states its license.

Citation: Chávez-Silverman, S. 2017. Black Holes-Deshielo Crónica. PORTAL Journal of Multidisciplinary International Studies, 14:1, 20-23. http:// dx.doi.org/10.5130/portal. v14i1.5355

ISSN 1449-2490 | Published by UTS ePRESS I http://portal. epress.lib.uts.edu.au
CULTURAL WORK

\section{Black Holes-Deshielo Crónica}

\section{Susana Chávez-Silverman}

Pomona College

Corresponding author: Professor Susana Chávez-Silverman, Romance Languages and Literatures, Pomona College, 333 N. College Way, Claremont CA 91711, USA. Suzanne.chavezsilvermanapomona.edu

DOI: http://dx.doi.org/10.5130/portal.v14i1.5355

Article History: Received 18/01/2017; Revised 14/03/2017; Accepted 15/03/2017; Published 04/05/2017

\section{Abstract}

Susana Chávez-Silverman is a Califas-born U.S. Latina writer and flaneuse who (against all odds) is also an educator, although she mostly laments what's happened to la cacademia over the last decade or so. She still finds joy and hope in being la High Priestess of SLOW to her students and former students, some of which are among her closest compinches. Of the two crónicas included in this special curated issue of cultural works in PORTAL under the theme Transitions and Dislocations, she says:

'My writing/life took a major detour in 2016. For most of the year, I wrote nothing at all. Or rather, nothing except e-mail after email: to administrators, colleagues all over the world, attorneys, friends and relatives. I had scheduled my sabbatical in order to finish a book project, but had been derailed by a Title IX investigation and plagiarism case at my home institution, and the accompanying anxiety and anger. I returned to my book over the summer, culling and editing hundreds of pages of primary texts (letters and emails). But this book is much longer-and truer-than anything l've written before and takes a different kind of writing energy, uninterrupted time, and commitment. Writing from a place of anger and anxiety doesn't serve this book. The two crónicas included in this special curated issue are not part of my book in progress, Our Ubuntu, Montenegro: del Balboa Café al Apartheid and Back. Rather, they were my way of easing back into my (helart space. 'Casi Víspera' proves I did write something in 2016 after all (other than email, I mean) - I had no memory of it until early this year! 'Black Holes' talks about precisely this (remembering and not). I was determined not to let 2016's plagiarism/Title IX toxic double helix continue to poison me. I wanted to begin 2017 with a bang, and wrote this for my son, for his 30th birthday.'

\section{Keywords}

\section{Susana Chávez-Silverman; crónica; chronicle}


U T S

e PRES S
1 enero, 2017

Claramonte, Califas

Con razón que I have lacunae. Big ol' black holes de la memoria. Subí just now para guardar las pages de la agenda del annus horribilis (2016) en el drawer del enorme escritorio que tengo arriba en el study. LITTLE EYE: nunca lo uso como tal一digo, as a desk, cual study. I just can't, ya que érase una vez it was my old bedroom, during my 100Year Sleep, o sea los 17 que pasé con el Dorian. Never mind que lo haya refaccionado after my Great Escape, painting the room a gorgeous Emerald City verde, installing fauxweathered, funky estantes and a jewel-red Ultrasuede sofa, plus ese enorme desk, antaño de Daddy, y antaño before him belonging to José F. Montesinos (el cuñado del mere mere Lorca, pero esa es otra ...). En el drawer, vi algunas otras agenda pages que tenía allí, guardadas.

Creo que nunca he mirado allí, I mean really looked; normally sólo guardo la agenda caduca y saco las new pages y así. A kind of New Year ritual. Pero a saber por qué, ahora me dio por mirar. There was 2004, and 2003. No sé cómo, pero en ese invisible linksy, azar objetivo, totalmente Cortázar kind of way, en una my gaze alighted on ...

\section{el 8 de febrero de 2003}

Until right now, esa fecha sólo me había significado algo beautiful y bittersweet: la famosa Last Night con mi Montenegro. Completely forgotten by me for many years, pero en 2008, en el Montalvo memory vortex, it had come back to me. I'd begun to remember. Todos pero todititos los minuciosos details de esa noche (que resultó no ser la last night after all y quizásshe says now, no sin una buena dosis de ironía - it should have been). Tan technicolor que al recordarlos—al recontártelos, Montenegro—I conjured you. I conjured us, de nuevo, a más de 25 años de distancia.

Tu cuerpo, nuestro sudor, a pesar de esa (so much colder back then, remember, na' que ver, poh, con este globally-overheated now) long, icy San Francisco night. Los restos de coca en mi hand mirror, las colillas en mi Cinzano ashtray, el que Daddy me había robado de ese outdoor café en Canarias, to mom's horror (aunque Daddy le aseguró mil veces, June, they're meant to be taken). Tu cuerpo, fornido y winter-pale (ningún rastro de sexy southern hemisphere bronze: tú y tus homies, el Du Toit y el Ken Steenkamp, ya llevaban semanas aquí en el norte by the time we met—you'd been white-washed, jaja), lanky and muscular. Our cold feet entwined, toda la noche.

Tu boca. Tu boca en la mía, en mi sexo. Your body above me, ah, tus hombros. Your wide, full-lipped mouth, soot-dark Capricorn eyes. Querías follarme como nadie, nunca, antes, you said. Oh the hubris, erotic and heartbreaking, de los 25 años.

Y acurrucados allí, horas y horas después (buzzed on coke y Remy Martin, on the lust and passion of our San Francisco idyll y la preemptive desesperación de tu next-day departure) we dozed off, finally, as the pearl-pale, opaque Marina dawn filtered through los matchstick slats de esos student-cheap bamboo blinds de Cost Plus.

Anygüey, como te estaba diciendo ... al subir al dizque study ahora, abrí el desk drawer donde guardo las pages de mis ex-agendas, para archivar las de 2016. Actually, no tengo guardadas tantos ex-años allí, I realized. En 2010, cuando mi fuga de Planet Dorian, I must've 
purged quite a few years' worth. Now, ni siquiera me acuerdo, ¿cuándo habré comenzado a llevar una agenda, anyway? Ni puta idea. Otro black hole...

Pero bueno: la cosa es que de repente (ni idea cómo, like I said: estas cosas me pasan), me eye fell upon some pages from 2004 (año que recordé, al tiro, como terribilis), then 2006, todo el año, which I decided to toss por aburrido (except la primera visita de Wim a Califas, that was epic, al pensarlo, y mi primer viaje a Puerto Rico para LASA, y luego a OZ ... si bien MAL acompañada por el sarcófago del Dorian, OB-vio ... uf).

A continuación, medio sticking together por los weird sticky restos de un ex-rubber band, vi algunas pages de 2003. Año hasta más terribilis, si cabe, que 2004. Pero I couldn't help myself. Ya había probado de ese magic mushroom, down ese rabbithole. I fanned those ancient agenda pages all around me, arrodillada allí en la beige sisal-look moqueta, y la primera que vi, distintiva e individual era-I promise you-el 8 de febrero, 2003.

No appointments, no horóscopos, no lunches, dinners, nada. Only one sentence written there:-you need to die, Mom. Pero oh ... 'twas enough (pace Mercutio): I was felled. Instantly.

Ojalá y pudiera decir que 'it all came back to me,' right then and there. Al leer esa odiosa, ominosa frase. Pero- a diferencia de los movies — así no trabaja la memoria. Al menos: not mine. Porque la verdad es que si bien he podido hacer memoria (oh, I still love this oh so 'Tine way of saying it - to remember-aun a 15 años de escucharlo en su habitat) del contexto histórico de la frase, I have no memory of what, specifically, provoked esa death wish ese día.

Porque ya había padecido más de un año de la outsized rabia del Juvenil, que comenzó en 2001, like clockwork, ni bien volvimos del año en Buenos Aires. Ese nuestro año porteño, I've seen it so clearly, poignantly since then-yo desarraigada allí pero re(dis)covering myself, becoming myself, my writerly self - también me compró un año extra de tu childhood, Joey. Vos, dislocado como yo, you'd sulked for the first few weeks, pero then you'd bloomed. Para cuando volvimos a la suburban college town reality de tu first year of high school, te habías adueñado de esa faraway city en el sur. Te habías hecho amigos, hablabas un español aporteñado. You didn't want to come back, ni yo tampoco.

Patrás en Califas, se te explotó una barroca American adolescencia con una double-dose de phallic, bad-ass, wannabe ghetto hostilidad. Which you aimed directo al solar plexus de lo que me era más caro, más yo: mi writing.

OJITO: Me tuve que interrumpir, just now, pa' llamar al Austra. Ostensibly pa' alertarle, cual Google alerta, jaja, de los five (in)visible links I'd just sent him, him y unos cuantos otros Tripulantes, on e-macho. I knew he'd be taken by la nota en el clove tree (pero 'really' on la globalización, las Spice Islas, la Dutch East India Co., la accidental colonización de la Américas y ... global warming) by Amitav Ghosh. Sabía que we'd bond, bigtime, over el Ghosh's rapsodias planteriles que al rato end up y te transportan a un lugar (o muchos) muy otro, completely unexpected. ¡Coño, que vato brillante!

Pero I said 'tuve que' porque me di cuenta, al estar platicando con el Austra, de que my heart was racing and I was breathing really fast. So, dije que no recuerdo (los detalles) and it's true, I don't. Pero the body remembers. Y hasta el Austra (not prone to emo-gushing) me dijo, cuando le leí la odiosa frase del Juvenil-god darls, that brings it all right back, doesn't it? I remember how intense and awful those days were for you. Entonces, fortificada por la breve plática planteril transpacífica con el Austra (who remembers), pude-puedo-volver a la brega. Get patrás en la montura and ride this love-bronco un little hair longer.

Anygüey Joey, te juro que even here, now, 14 years en el futuro de esa rage-fuelled missile, no me viene a la memoria su por qué. $Y$ ¿sabes qué? Maybe it's better this way. Porque vos te 
acordás. De eso, estoy segura. In the TRC of our shared heart—lo más hermoso de este wack, espantoso año pasado, añito del fucking Chinese Chango, for which I'd had such high hopeste me has disculpado. Estoy casi segura de que you even specifically recalled that very deathwish. Y me dijiste que it wasn't about me. Que nada que ver conmigo. Que it was all about you. Que eran cosas que hubieses querido decir a tu dad, pero ... no te atrevías and he was nowhere to be found en esos días (well ni entonces ni now, pero ... esa es otra). Pero el punto de todo esto es que creo que los black holes no son, necesariamente—o no siempre—a bad thing.

El máximo recuerdo del 8 de febrero—el original, de 1982—had been glacier-buried más de 25 años cuando comenzó el thaw-el memory-melt-en 2008, en el Montalvo Arts Center. Y ese deshielo, esa recovery, por destabilizing que fuera (y me terremoteó, but good, el concepto de mi pasado, de mí, de quién yo era), it was a gift. Porque me devolvió a Montenegro. Y eso (aunque eventualmente te volvieras a tu Southforky, sleepwalking life, oh Rip van Winkle)— ese return—me despertó de mi propia Bella Durmiente 100-year sleep. Y de esa stark, lúcida vigilia — awake, finally, al horror de mi daily (muerte en) vida y a la urgencia de cambiarla— nunca más me volví a dormir.

Aunque me tomara un par de añitos más, esa nueva vigilia (que me había devuelto intactos y nítidos los recuerdos-oh so lushly embodied—de ese original 8 de febrero, y de tantas otras San Francisco and New Orleans nights, de soñolientas y eróticas Houston mornings y de nuestra helada Mardi Gras despedida en Chicago) me fortaleció para emprender mi Great Escape en septiembre de 2010.

Así que ¿the moral of the story, m'ijito? Let's lance ourselves forward onto the spear, como dice el Sokoloff. Live with the question. Abracemos, in other words, las operaciones selectivas de la memoria. Porque he aquí su razón de ser: a veces el olvido es balsámico, como cuando miré esa agenda page —el 8 de febrero, 2003 — and had not a fucking clue why you'd said what you'd said. Seguro que hay algo allí dark and ugly, deep down below. Pero ¿¿sabes qué? En este caso, mejor que sigan roncando esos sleeping canes. You and I are in a good place now y como dije antes, I think your own remembering is enough for the both of us, ¿que no?

Por otra parte, cuando hay algo glacier-buried que te podría cambiar la vida para bien-like it saved my life - pos órale. Esa es otra cosa. Lance yourself. Go for it. Al comienzo de un nuevo año, el Año del Fire Phoenix (mejor que el Roostie, ¿no te parece?)—your 30 $30^{\text {th }}$ year, coño—celebremos este thaw. 